\title{
High Tibial Osteotomy versus Unicompartmental Knee Arthroplasty for Medial Compartment Arthrosis with Kissing Lesions in Relatively Young Patients
}

\author{
Seung Min Ryu, MD, Jae Woo Park, MD, Ho Dong Na, MD, and Oog Jin Shon, MD \\ Department of Orthopedic Surgery, Yeungnam University Medical Center, Daegu, Korea
}

\begin{abstract}
Purpose: The purpose of this study is to compare the clinical and radiographic outcomes of high tibial osteotomy (HTO) and unicompartmental arthroplasty (UKA) in advanced medial compartment arthritis accompanied by kissing lesions in relatively young patients.

Materials and Methods: Forty-five patients were divided into the HTO $(n=23)$ and UKA $(n=22)$ groups. Clinically, we evaluated the Lysholm knee scoring scale, visual analogue scale, Hospital for Special Surgery, and Western Ontario and McMaster Universities Osteoarthritis index scores preoperatively, 6 and 12 months postoperatively, and at the final follow-up. Radiographically, we measured the femoral-tibial angle and mechanical axis deviation preoperatively and at the final follow-up.

Results: All clinical outcomes gradually improved in both groups from the postoperative period to the final follow-up. At the final follow-up, all clinical outcomes were slightly better in the UKA group than in the HTO group; however, differences were not statistically significant.

Conclusions: HTO is comparable to UKA in terms of clinical outcomes. Thus, the results of this study suggest that HTO might be a good alternative treatment to UKA for medial unicompartmental arthritis accompanied by kissing lesions in relatively young patients.
\end{abstract}

Keywords: Knee, Osteoarthritis, Osteotomy, Arthroplasty, Unicompartmental

\section{Introduction}

The treatment for unicompartmental osteoarthritis (OA) in young patients is still a controversial issue. The possible treatment methods of this disease are non-operative or operative treatment; operative treatment can be divided into arthroscopy, high tibial osteotomy (HTO), and unicompartmental arthroplasty (UKA $)^{1-3)}$. However, the treatment outcomes of these surgical treatment modalities for unicompartmental OA have become subjects of controversy.

Received February 3, 2017; Revised (1st) March 11, 2017;

(2nd) May 4, 2017; (3rd) June 20, 2017;

Accepted June 26, 2017

Correspondence to: Oog Jin Shon, MD

Department of Orthopedic Surgery, Yeungnam University Medical

Center, 170 Hyeonchung-ro, Nam-gu, Daegu 42415, Korea

Tel: +82-53-620-3640, Fax: +82-53-628-4020

E-mail: maestro-jin@hanmail.net

This is an Open Access article distributed under the terms of the Creative Commons Attribution Non-Commercial License (http://creativecommons.org/licenses/by-nc/4.0/) which permits unrestricted non-commercial use, distribution, and reproduction in any medium, provided the original work is properly cited.
The ideal indication for HTO is young ( $<65$ years old), active patients affected by symptomatic mild to moderate varus knee (around $10^{\circ}$ ) with medial compartment involvement (less than grade III Ahlback classification), good knee range of motion, no severe knee instability, and mild meniscus injury ${ }^{4-6)}$. On the other hand, UKA is ideal if the patients are $>60$ years old and not obese and have low activity demand and unicompartmental femoral or tibial condyle avascular necrosis ${ }^{7,8)}$. However, only a small proportion of patients can be considered eligible for either HTO or UKA; they are the so-called borderline patients ${ }^{9,10)}$. The indications for both HTO and UKA include ages 55 to 65 years, moderate activity, mild varus alignment, less than grade I knee instability, and Ahlback grades I to II arthrosis.

In cases of advanced medial compartment arthritis accompanied by kissing lesions, HTO is known to be a relative contraindication, and UKA is preferred instead ${ }^{11)}$. However, in relatively young patients, UKA or total knee arthroplasty (TKA) can be disastrous when complications, such as component loosening, failure, and infection occur ${ }^{7}$. Furthermore, revisions of UKA and TKA in younger patients have shown poor outcomes ${ }^{12}$. On the 
other hand, some studies have reported that HTO combined with arthroscopic microfracture in young patients with unicompartmental arthritis with kissing lesions can be effective in alleviating pain and in preserving the joint in a short-term follow-up ${ }^{13-15)}$.

However, to the best of our knowledge, no previous study comparing the outcomes of HTO and UKA in young patients with unicompartmental arthritis with kissing lesions has been reported. Hence, the purpose of this study was to compare the clinical and radiographic outcomes between HTO and UKA in relatively young patients with unicompartmental arthritis with kissing lesions.

\section{Materials and Methods}

\section{Patient Selection}

This study was approved by the Institutional Review Board of our hospital. From January 2007 to January 2012, all patients who were treated with HTO or UKA and had medial compartmental arthritis with kissing lesions were reviewed in this study. The kissing lesions affected both the contacting femoral and tibial condyles; these were diagnosed when the femoral condyle and tibial condyle were in contact on $45^{\circ}$ flexion weight-bearing posterior-anterior radiographs (Fig. 1). Intraoperatively, these were classified as Outerbridge grade IV lesions that appeared to be in contact with each other in the femoral condyle and tibial condyle. Of 60 patients in total, seven patients whose follow-up were $<2$ years, three who had lateral meniscus lesions or lateral compartment arthritis, three who had insufficiencies in the an-

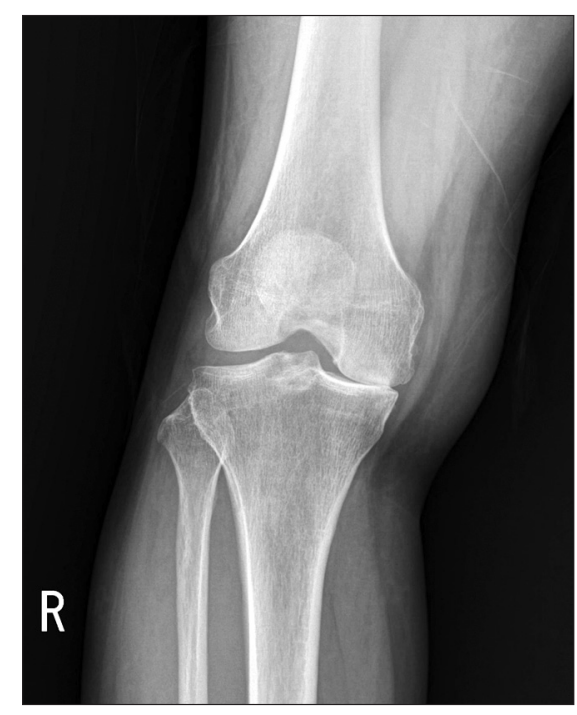

Fig. 1. A 54-year-old female who had medial compartmental arthritis combined with a kissing lesion. The kissing lesion was diagnosed when the femoral condyle and tibial condyle were seen in contact on the $45^{\circ}$ flexion weight bearing posterior-anterior radiograph. terior or posterior cruciate ligaments, and two who had severe patellofemoral OA were excluded. No patient who had a deformity or history of trauma in any limb was included to evaluate the accurate alignment of the lower limbs. Ultimately, 45 patients were divided into the HTO group ( $\mathrm{n}=23)$ and UKA group $(\mathrm{n}=22)$ for a comparative analysis (Table 1). Age, sex, and mean followup period were not statistically significantly different; however, body mass index (BMI) showed a statistically significant difference between the two groups $(\mathrm{p}=0.020)$. The patients were asked to decide whether to undergo either HTO or UKA after a discussion with the surgeon about the prognosis, rehabilitation program, and their expectation of the level of physical activity after surgery. Based on the evaluation of the patient's compliance, their ability to reduce weight, and hygiene, we finally decide proper treatment. HTO was recommended for patients with severe labor, such as farming, and UKA was recommended for sedentary patients in urban areas.

\section{Surgical Technique}

In the HTO group, a longitudinal skin incision was created at the medial side of the tibial tuberosity. The superficial medial collateral ligament was completely released below the osteotomy site. Biplanar medial open wedge osteotomy was performed using the ChronOS vivify spacer (DePuy Synthes, Solothurn, Switzerland) and TomoFix plate (DePuy Synthes). The target correction angle was measured at the point where the mechanical axis of the lower limb passed through the Fujisawa point, which was $62.5 \%$ from the medial tibial articular margin ${ }^{16)}$.

In the UKA group, a standard medial parapatellar arthrotomy was performed. UKA was performed without extension to the

Table 1. Patient Demographics

\begin{tabular}{lccc}
\hline \multicolumn{1}{c}{ Variable } & HTO group & UKA group & p-value \\
\hline Age $(\mathrm{yr})$ & $57.6 \pm 6.4$ & $60.5 \pm 3.4$ & $0.121^{\mathrm{a})}$ \\
Sex $(\mathrm{M}: \mathrm{F})$ & $2: 21$ & $3: 19$ & $0.665^{\mathrm{b})}$ \\
BMI $\left(\mathrm{kg} / \mathrm{m}^{2}\right)$ & $27.7 \pm 2.9$ & $25.4 \pm 3.6$ & $0.020^{\mathrm{c})}$ \\
FTA $\left({ }^{\circ}\right)^{\mathrm{d})}$ & $-4.3 \pm 3.2$ & $0.8 \pm 3.4$ & $0.000^{\mathrm{a})}$ \\
MAD (\%) & $8.4 \pm 17.2$ & $16.0 \pm 15.5$ & $0.126^{\mathrm{a})}$ \\
Mean follow-up (mo) & $40.0 \pm 19.4$ & $33.1 \pm 8.7$ & $0.400^{\mathrm{a})}$ \\
\hline
\end{tabular}

Values are presented as mean \pm standard deviation.

HTO: high tibial osteotomy, UKA: unicompartmental arthroplasty, BMI: body mass index, FTA: femoral-tibial angle, MAD: mechanical axis deviation.

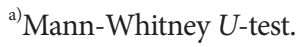

${ }^{b)}$ Fisher exact test.

${ }^{c}$ Student $t$-test.

${ }^{\mathrm{d})}$ Varus is defined as minus; valgus is defined as plus. 
vastus medialis obliquus and without patella eversion. The sigma unicompartmental knee prostheses (DePuy, Warsaw, IN, USA) were applied in all patients in the UKA group.

\section{Evaluation Tools}

Clinically evaluation was performed using the Lysholm knee scoring scale ${ }^{17)}$, visual analogue scale ${ }^{18)}$, Hospital for Special Surgery $(\mathrm{HSS})^{19}$, and Western Ontario and McMaster Universities Osteoarthritis index scores ${ }^{20)}$ preoperatively, 6 and 12 months postoperatively, and at the final follow-up.

We radiographically measured the femoral-tibial angle (FTA) and mechanical axis deviation (MAD) preoperatively and at the final follow-up (Fig. 2). Negative FTA and MAD values were given to the knees in varus alignment.

To determine the intra- and interobserver reliabilities of the radiographic outcomes, two observers (SMR and HDN) evaluated 20 randomly selected radiographs regardless of HTO or UKA that were included in this series. The two observers were blinded to patient information and independently measured the preoperative and postoperative FTA and MAD values of the radiographs
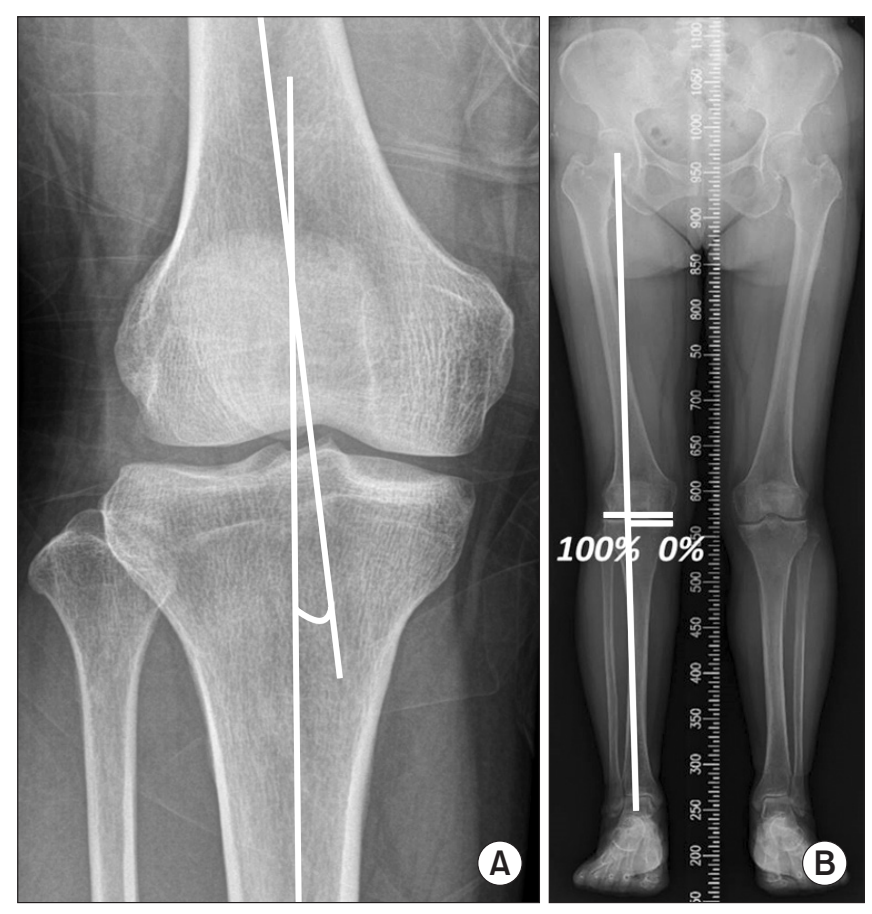

Fig. 2. (A) Femoral-tibial angle was defined as the angle between the anatomical axis of the femur and the tibia in the anteroposterior (AP) view of the knee joint. (B) For the assessment of mechanical axis deviation (MAD), a line was drawn from the center of the ankle joint to the head of the femur through that of the knee joint on the weight bearing full-limb AP radiograph. MAD was calculated as the horizontal distance from this line to the medial edge of the tibial plateau divided by the total width of the tibial plateau. and repeated the measurements 3 weeks later. The intra- and interobserver reliabilities were analyzed using intraclass correlation coefficients (ICCs) with $95 \%$ confidence intervals.

We also compared the complications after surgery between the two procedures, such as fixation failure, intra-articular fracture, infection, and component loosening or breakage.

\section{Statistical Analysis}

Power calculations for sample size were performed using the $\mathrm{G}^{\star}$ Power program ${ }^{211}$. The sample size was based on the results reported by Ivarsson and Gillquist ${ }^{22}$. The mean Lysholm knee scoring scale scores of the HTO and UKA groups were 78 and 91, respectively, and their corresponding standard deviations were 19 and 11 , respectively. With a power of $0.8,20$ cases for each group were needed for this study.

All statistical analyses were performed using the IBM SPSS ver. 23.0 (IBM Co., Armonk, NY, USA), and a p-value of $<0.05$ was considered statistically significant. The Mann-Whitney $U$-test, Fisher exact test, and Student $t$-test were used to show statistical differences between the two groups. Wilcoxon signed rank test was also used to compare radiographic values between preoperative and final follow-up assessments.

\section{Results}

\section{Clinical Outcomes}

The clinical outcomes are shown in Table 2. All clinical outcomes gradually improved in both groups from the postoperative period to the final follow-up. At the postoperative 6 months, Lysholm knee scoring scale and HSS score showed better result in UKA group, and difference was statistically significant ( $\mathrm{p}=0.032$ and $\mathrm{p}=0.041$ ). However, all clinical outcomes between the two groups showed no significant differences statistically at the postoperative 1 year and the final follow-up ( $p>0.05)$.

\section{Radiographic Outcomes}

The radiographic outcomes are shown in Tables 1 and 3. The mean FTA was $-4.3^{\circ}$ and $0.8^{\circ}$ in the HTO and UKA groups, respectively, before surgery, and the difference was statistically significant $(\mathrm{p}=0.000)$. At the final follow-up, the mean FTA was $7.8^{\circ}$ and $5.6^{\circ}$ in the two groups, respectively. The mean MAD was $8.4 \%$ and $16.0 \%$, respectively, before surgery, and the difference was not statistically significant $(\mathrm{p}=0.126)$. However, at the final follow-up, the mean MAD was $56.7 \%$ and $44.5 \%$, respectively. The differences from the preoperative assessment to the final followup in both groups were all statistically significant (all $\mathrm{p}=0.000$ ). 
Table 2. Clinical Outcomes of the HTO Group and UKA Group

\begin{tabular}{lcccc}
\hline Group & Preoperative & $\begin{array}{c}\text { POD } \\
6 \mathrm{mo}\end{array}$ & $\begin{array}{c}\text { POD } \\
1 \mathrm{yr}\end{array}$ & $\begin{array}{c}\text { Final } \\
\text { follow-up }\end{array}$ \\
\hline Lysholm score & & & & \\
HTO & $50.2 \pm 15.7$ & $81.4 \pm 14.6$ & $86.6 \pm 13.9$ & $87.4 \pm 12.0$ \\
UKA & $51.4 \pm 7.7$ & $85.2 \pm 9.3$ & $88.2 \pm 9.9$ & $89.2 \pm 10.2$ \\
p-value & 0.641 & 0.032 & 0.937 & 0.445 \\
VAS score & & & & \\
HTO & $6.5 \pm 1.2$ & $3.8 \pm 1.5$ & $3.2 \pm 1.5$ & $2.2 \pm 1.2$ \\
UKA & $6.4 \pm 1.0$ & $3.2 \pm 1.4$ & $3.1 \pm 1.8$ & $1.5 \pm 1.7$ \\
p-value & 0.194 & 0.889 & 0.482 & 0.303 \\
HSS score & & & & \\
HTO & $55.6 \pm 13.1$ & $80.1 \pm 7.3$ & $85.3 \pm 6.6$ & $85.1 \pm 8.3$ \\
UKA $_{\text {p-value }}$ & $55.0 \pm 9.0$ & $84.1 \pm 8.3$ & $86.3 \pm 7.3$ & $86.6 \pm 7.9$ \\
WOMAC score $_{\text {a) }}$ & 0.520 & 0.041 & 1.000 & 0.625 \\
HTO $_{\text {UKA }}$ & $57.0 \pm 22.9$ & $28.2 \pm 17.2$ & $18.6 \pm 19.1$ & $16.5 \pm 17.5$ \\
p-value $^{\text {a) }}$ & $63.2 \pm 18.3$ & $24.0 \pm 18.3$ & $16.5 \pm 18.8$ & $14.9 \pm 16.9$ \\
\hline
\end{tabular}

Values are presented as mean \pm standard deviation.

HTO: high tibial osteotomy, UKA: unicompartmental arthroplasty, POD: postoperative day, Lysholm: Lysholm knee scoring scale, VAS: visual analogue scale, HSS: Hospital for Special Surgery, WOMAC: Western Ontario and McMaster Universities Osteoarthritis index.

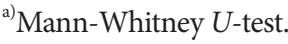

Both intra- and interobserver reliability testing results were 0.8 (Table 4). The ICC values of MAD were greater than those of FTA for both intra- and interobserver reliabilities.

\section{Complications}

No specific complications were observed during the 1-year short-term follow-up period in both groups.

\section{Discussion}

This study was conducted to compare the results of HTO and UKA for medial unicompartmental knee arthritis accompanied by kissing lesions in relatively young patients. There were no significant differences in the clinical outcomes between the two treatment methods at the final follow-up. However, the radiographic outcomes showed that HTO tended to correct a greater angle than UKA.

Numerous studies reported the clinical outcomes of HTO and UKA in unicompartmental arthritis ${ }^{23,24)}$. Although these studies did not compare such conditions accompanied by kissing lesions,
Table 3. Radiographic Outcomes of the HTO Group and UKA Group

\begin{tabular}{cccc}
\hline Group & Preoperative & Final follow-up & p-value ${ }^{\mathrm{b})}$ \\
\hline FTA $\left({ }^{\circ}\right)^{\mathrm{a})}$ & & & \\
HTO & $-4.3 \pm 3.2$ & $7.8 \pm 2.7$ & 0.000 \\
UKA & $0.8 \pm 3.4$ & $5.6 \pm 1.9$ & 0.000 \\
MAD $(\%)^{\mathrm{a})}$ & & & \\
HTO & $8.4 \pm 17.2$ & $56.7 \pm 12.2$ & 0.000 \\
UKA & $16.0 \pm 15.5$ & $44.5 \pm 13.8$ & 0.000 \\
\hline
\end{tabular}

Values are presented as mean \pm standard deviation.

HTO: high tibial osteotomy, UKA: unicompartmental arthroplasty, FTA: femoral-tibial angle, MAD: mechanical axis deviation.

${ }^{\text {a) }}$ Varus is defined as minus; valgus is defined as plus.

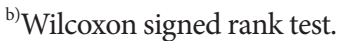

Table 4. Intraobserver and Interobserver Reliabilities of the Radiographic Evaluations

\begin{tabular}{lcc}
\hline & $\left.\mathrm{FTA}^{\circ}\right)$ & $\operatorname{MAD}(\%)$ \\
\hline Intraobserver reliability & $0.946(0.844-0.985)$ & $0.977(0.956-0.985)$ \\
Interobserver reliability & $0.892(0.687-0.971)$ & $0.901(0.728-0.968)$ \\
\hline
\end{tabular}

Values are presented as intraclass correlation coefficients (95\% confidence interval).

FTA: femoral-tibial angle, MAD: mechanical axis deviation.

the differences in the clinical outcomes were not statistically significant between the two procedures. Similarly, the clinical outcomes of this study showed no statistically significant difference between HTO and UKA in the cases accompanied by kissing lesions at the final follow-up.

On the other hand, there are several reports that clinical outcome of UKA is better in unicompartmental arthritis less than 1 year follow up after surgery. Ivarsson and Gillquist ${ }^{22)}$ reported that UKA group showed better results than HTO group at 6 months postoperatively. And Borjesson et al. ${ }^{25)}$ also reported that time-distance variables of gait showed clinically significant differences in favor of the UKA group at 3 months postoperatively. Our results also showed that UKA group showed better results than HTO group at 6 months postoperatively in Lysholm and HSS score with statistically significance.

Only a few studies have directly compared the radiographic outcomes of HTO and UKA in unicompartmental arthritis ${ }^{26,27}$. In general, reported radiographic outcomes showed no statistically significant difference between HTO and UKA. However, the mean MADs of HTO and UKA of this study were $56.7 \%$ and $44.5 \%$, respectively, and HTO was closer to the Fujisawa point ${ }^{16}$. We speculate that the differences in the radiographic data were 
because of the surgeon's own target angle, which was different between the two surgical methods.

HTO was once known as a relative contradiction for patients with advanced arthritis accompanied by a kissing lesion, and arthroplasty is considered to offer more favorable outcome in most cases combined with kissing lesions ${ }^{11}$. However, there are numerous patients to which knee arthroplasty can be hardly applied owing to the risk of infection and the difficulty in management. Although there was no major complication in the UKA group in our study, if complications occur, such as component loosening or infection, it is disastrous for young patients as revision operations are more difficult. Extended life expectancy has made patients more unwilling to undergo knee revision arthroplasty, and a long-term follow-up has found that UKA is not effective in every case ${ }^{28-30)}$. In our study, although all clinical outcomes were slightly better in the UKA group than in the HTO group, there was no statistically significant difference. Therefore, we suggest that HTO might be a good alternative treatment for medial unicompartmental arthritis with kissing lesions in relatively young, borderline patients considering the risk of arthroplasty.

The main limitations of this study are that it was a retrospective study, and the number of cases was relatively small. Furthermore, the duration of follow-up was relatively short. Since the differences in both preoperative BMI and FTA between the two groups were statistically significant, it is presumed that an accurate comparison between the two groups could not be evaluated. Moreover, as the clinical outcomes between the two groups showed nonparametric values, we could not perform a repeated measures analysis of variance (ANOVA) analysis; thus, we could not evaluate the interaction between the time and treatment method. However, to the best of our knowledge, this is the first study to compare the clinical and radiographic outcomes of HTO and UKA in medial compartmental arthritis in relatively young patients with kissing lesions.

\section{Conclusions}

HTO was comparable to UKA in terms of clinical outcomes and complications in unicompartmental arthritis accompanied by kissing lesions in relatively young, borderline patients. Thus, the results of this study suggest that HTO might be a good alternative treatment to UKA for medial unicompartmental arthritis with kissing lesions in this type of patient population.

\section{Conflict of Interest}

No potential conflict of interest relevant to this article was reported.

\section{References}

1. Billings A, Scott DF, Camargo MP, Hofmann AA. High tibial osteotomy with a calibrated osteotomy guide, rigid internal fixation, and early motion: long-term follow-up. J Bone Joint Surg Am. 2000;82:70-9.

2. Murphy SB. Tibial osteotomy for genu varum. Indications, preoperative planning, and technique. Orthop Clin North Am. 1994;25:477-82.

3. Weale AE, Lee AS, MacEachern AG. High tibial osteotomy using a dynamic axial external fixator. Clin Orthop Relat Res. 2001;(382):154-67.

4. Naudie D, Bourne RB, Rorabeck CH, Bourne TJ. The Install Award: survivorship of the high tibial valgus osteotomy: a 10- to -22-year followup study. Clin Orthop Relat Res. 1999; (367):18-27.

5. Preston S, Howard J, Naudie D, Somerville L, McAuley J. Total knee arthroplasty after high tibial osteotomy: no differences between medial and lateral osteotomy approaches. Clin Orthop Relat Res. 2014;472:105-10.

6. Naudie DD, Amendola A, Fowler PJ. Opening wedge high tibial osteotomy for symptomatic hyperextension-varus thrust. Am J Sports Med. 2004;32:60-70.

7. Kozinn SC, Scott R. Unicondylar knee arthroplasty. J Bone Joint Surg Am. 1989;71:145-50.

8. Borus T, Thornhill T. Unicompartmental knee arthroplasty. J Am Acad Orthop Surg. 2008;16:9-18.

9. Dettoni F, Bonasia DE, Castoldi F, Bruzzone M, Blonna D, Rossi R. High tibial osteotomy versus unicompartmental knee arthroplasty for medial compartment arthrosis of the knee: a review of the literature. Iowa Orthop J. 2010;30:13140.

10. Rossi R, Bonasia DE, Amendola A. The role of high tibial osteotomy in the varus knee. J Am Acad Orthop Surg. 2011; 19:590-9.

11. Bonasia DE, Dettoni F, Sito G, Blonna D, Marmotti A, Bruzzone M, Castoldi F, Rossi R. Medial opening wedge high tibial osteotomy for medial compartment overload/arthritis in the varus knee: prognostic factors. Am J Sports Med. 2014; 42:690-8.

12. Hang JR, Stanford TE, Graves SE, Davidson DC, de Steiger 
RN, Miller LN. Outcome of revision of unicompartmental knee replacement. Acta Orthop. 2010;81:95-8.

13. Pascale W, Luraghi S, Perico L, Pascale V. Do microfractures improve high tibial osteotomy outcome? Orthopedics. 2011; 34:e251-5.

14. van den Bekerom MP, Patt TW, Rutten S, Raven EE, van de Vis HM, Albers GH. Arthroscopic debridement for grade III and IV chondromalacia of the knee in patients older than 60 years. J Knee Surg. 2007;20:271-6.

15. Shon OJ, Park SJ, Shim BJ, Lee DY. Comparative study of clinical and radiographic outcomes of high tibial osteotomy in patients with kissing lesions and non-kissing lesions. Knee Surg Relat Res. 2017;29:288-94.

16. Fujisawa Y, Masuhara K, Shiomi S. The effect of high tibial osteotomy on osteoarthritis of the knee: an arthroscopic study of 54 knee joints. Orthop Clin North Am. 1979;10:585608.

17. Lysholm J, Gillquist J. Evaluation of knee ligament surgery results with special emphasis on use of a scoring scale. Am J Sports Med. 1982;10:150-4.

18. Carlsson AM. Assessment of chronic pain. I. Aspects of the reliability and validity of the visual analogue scale. Pain. 1983;16:87-101.

19. Insall JN, Dorr LD, Scott RD, Scott WN. Rationale of the Knee Society clinical rating system. Clin Orthop Relat Res. 1989;(248):13-4.

20. Bellamy N, Buchanan WW, Goldsmith CH, Campbell J, Stitt LW. Validation study of WOMAC: a health status instrument for measuring clinically important patient relevant outcomes to antirheumatic drug therapy in patients with osteoarthritis of the hip or knee. J Rheumatol. 1988;15:1833-40.

21. Erdfelder E, Faul F, Buchner A. GPOWER: a general power analysis program. Behav Res Methods Instrum Comput. 1996;28:1-11.

22. Ivarsson I, Gillquist J. Rehabilitation after high tibial oste- otomy and unicompartmental arthroplasty: a comparative study. Clin Orthop Relat Res. 1991;(266):139-44.

23. Nwachukwu BU, McCormick FM, Schairer WW, Frank RM, Provencher MT, Roche MW. Unicompartmental knee arthroplasty versus high tibial osteotomy: United States practice patterns for the surgical treatment of unicompartmental arthritis. J Arthroplasty. 2014;29:1586-9.

24. Yim JH, Song EK, Seo HY, Kim MS, Seon JK. Comparison of high tibial osteotomy and unicompartmental knee arthroplasty at a minimum follow-up of 3 years. J Arthroplasty. 2013;28:243-7.

25. Borjesson M, Weidenhielm L, Mattsson E, Olsson E. Gait and clinical measurements in patients with knee osteoarthritis after surgery: a prospective 5-year follow-up study. Knee. 2005;12:121-7.

26. Takeuchi R, Umemoto Y, Aratake M, Bito H, Saito I, Kumagai K, Sasaki Y, Akamatsu Y, Ishikawa H, Koshino T, Saito T. A mid term comparison of open wedge high tibial osteotomy vs unicompartmental knee arthroplasty for medial compartment osteoarthritis of the knee. J Orthop Surg Res. 2010;5:65.

27. Stukenborg-Colsman C, Wirth CJ, Lazovic D, Wefer A. High tibial osteotomy versus unicompartmental joint replacement in unicompartmental knee joint osteoarthritis: 7-10-year follow-up prospective randomised study. Knee. 2001;8:18794.

28. W-Dahl A, Robertsson O, Lidgren L. Surgery for knee osteoarthritis in younger patients. Acta Orthop. 2010;81:161-4.

29. Warme BA, Aalderink K, Amendola A. Is there a role for high tibial osteotomies in the athlete? Sports Health. 2011;3: 59-69.

30. Mancuso F, Dodd CA, Murray DW, Pandit H. Medial unicompartmental knee arthroplasty in the ACL-deficient knee. J Orthop Traumatol. 2016;17:267-75. 